\title{
REDESCOBERTA DE ANATHALLIS BREVIPES (ORCHIDACEAE) NO ESTADO DE SÃO PAULO, BRASIL
}

\author{
GABRIEL MENDES MARCUSSOํ \& PABLO RODRIGUES SANINE²
}

Summary: Marcusso, G. M. \& P. Rodrigues Sanine. 2016. Rediscovery of Anathallis brevipes (Orchidaceae) in São Paulo State, Brazil. Bonplandia 25(1): 53-57.

This is the report of the rediscovery of Anathallis brevipes (H. Focke) Pridgeon \& M.W. Chase in Botucatu municipality, São Paulo State, Brazil. The last record in the state was reported more than 50 years ago. This new record shows the gaps of knowledge in the State and demonstrates the floristic importance of the "Cuestas" region.

Key words: Atlantic Forest, Cuestas region, Pleurothallidinae.

Resumo: Marcusso, G. M. \& P. Rodrigues Sanine. 2016. Redescoberta de Anathallis brevipes (Orchidaceae) no estado de São Paulo, Brasil. Bonplandia 25(1): 53-57.

Esse trabalho relata a redescoberta de Anathallis brevipes (H. Focke) Pridgeon \& M.W. Chase no município de Botucatu, estado de São Paulo, Brasil. A última coleta para o estado foi realizada há mais de 50 anos. Este novo registro demonstra a existência de lacunas de conhecimento no estado, assim como demonstra a importância florística da região das Cuestas.

Palavras chave: Cuestas, Floresta Atlântica, Pleurothallidinae.

\section{Introdução}

Anathallis Barb. Rodr. anteriormente incluso em Pleurothallis R. Br. sensu lato pertence à subtribo Pleurothallidinae. Distingue-se dos gêneros segregados de Pleurothallis s.l., como Acianthera Scheidw., Pabstiella Brieger \& Senghas e Specklinia Lindl. por apresentar as sépalas livres e de Myoxanthus Poeppig \& Endlicher por apresentar as bainhas do ramicaule glabras (Chiron \& Bolsanello, 2015). O gênero apresenta cerca de 120 espécies, ocorrendo desde o sul do México até o Brasil e Argentina (Pridgeon et al., 2005). No Brasil, o gênero está representado por 94 espécies, sendo 78 endêmicas do país, 36 ocorrem em São Paulo (Barros et al., 2015).

Anathallis brevipes (H. Focke) Pridgeon \& M.W. Chase é uma das que ocorrem no Estado de São Paulo, porém, é conhecida por uma única coleta (A. Gehrt s.n., SP 31582) que foi realizada em 1934, no município de Itu. A ausência de coletas por mais de 50 anos é um dos critérios do Livro Vermelho das Espécies Ameaçadas do Estado de São Paulo (Mamede et al., 2007), contudo, o táxon não se encontra listado. Uma das justificativas é que Pleurothallis s.l. não foi totalmente estudado

\footnotetext{
${ }^{1}$ Universidade Estadual Paulista "Júlio de Mesquita Filho", Instituto de Biologia, Departamento de Botânica, Av. 24 A, CEP: 13506-000, Rio Claro, SP. E-mail: gabrielmarcusso@hotmail.com

${ }^{2}$ Secretaria de Estado de Educação, SEE, Diretoria de Ensino Região de Botucatu, SP. Brasil. E-mail: sanineff@yahoo. com.br
} 
no estado, e portanto, ainda há lacunas sobre seu real status (Barros, 2007).

O presente trabalho tem como objetivos registrar a redescoberta de A. brevipes após mais de 50 anos no Estado de São Paulo, apresentar breve caracterização morfológica, comentários ecológicos e discutir o status de conservação.

\section{Material e Métodos}

A redescoberta foi feita no município de Botucatu (2255'23'S, 48²7'28'W), que se situa na província geomorfológica do Planalto da Bacia do Paraná, entre a Cuesta e a Depressão Periférica (Ponçano etal., 1981) (Fig. 1). Aregião é caracterizada como um ecótono entre dois "hotspots" brasileiros: Cerrado e Mata Atlântica (Myers et al., 2000). O local onde o espécime foi registrado apresenta a fitofisionomia da Floresta Estacional Semidecídua (IBGE, 2012) e o clima da região é, segundo a classificação de Köeppen, tropical de altitude (Cwa), com chuva no verão e seca no inverno. A precipitação média anual é de $1.358,6 \mathrm{~mm}$ e a média anual da temperatura é de $20,7^{\circ} \mathrm{C}$ (Cepagri, 2015).

O espécime foi coletado estéril e cultivado até a floração, posteriormente foi realizada a identificação e a incorporação no acervo do Herbário Rioclarense (HRCB) da Universidade Estadual Paulista, Rio Claro, SP.

\section{Resultados e Discussão}

Anathallis brevipes (H. Focke) Pridgeon \& M.W. Chase; Lindleyana 16(4): 247-248. 2001. Typus: Suriname: Hab. in sylvis ad rivum Para, supra arbores, Willschlaegel 1589 (W). Fig. 2.

Pleurothallis brevipes H. Focke, Tijdschr. Natuurk. Wetensch. Kunsten 2: 198. 1849.

Lepanthes modesta Barb. Rodr., Gen. Spec. Orchid. 2: 62. 1881.

Humboltia brevipes (H. Focke) Kuntze, Revis. Gen. P1. 2: 667. 1891.

Pleurothallis modesta (Barb. Rodr.) Cogn. in C.F.P. von Martius \& auct. suc. (eds.), Fl. Bras. 3(4): 449. 1896.
Anathallis modesta (Barb. Rodr.) Pridgeon \& M.W. Chase, Lindleyana 16: 249. 2001.

Specklinia bradei (H. Focke) Luer, Monogr. Syst. Bot. Missouri Bot. Gard. 95: 259. 2004.

Specklinia modesta (Barb. Rodr.) Luer, Monogr. Syst. Bot. Missouri Bot. Gard. 95: 262. 2004.

Panmorphia brevipes (H. Focke) Luer, Monogr. Syst. Bot. Missouri Bot. Gard. 105: 148. 2006.

Erva epífita, com 7-13 cm de altura; rizoma 1-1,3 mm de comprimento; ramicaule cilíndrico $2-7 \mathrm{~cm}$ de comprimento, coberto por bainhas; folha elíptico-oblonga com ápice tridentado; inflorescências em racemos múltiplos, curtos, não ultrapassando a altura das folhas, raque fractiflexa, saindo da base da folha; ovário 1,3 mm de comprimento, vináceo com faixas esbranquiçadas; flores eretas, paucifloras; sépalas laterais lanceoladas a elípticas, brevemente acuminadas, falciformes, 3-5 $\times 1-3 \mathrm{~mm}$, e a dorsal lanceolada, $5 \times$ 1,5-2 $\mathrm{mm}$, ambas pubescentes e vináceas com a base branca; pétalas lanceoladas, falciformes, acuminadas, com margem pilosa, $3 \times 0,8 \mathrm{~mm}$, vináceas; labelo ereto, brevemente unguiculado, articulado, oblongo, base com dois lobos e ápice arredondado, margem pilosa, vináceo, $3 \times 0,5 \mathrm{~mm}$; coluna claviforme, clinândrio tridentado com margem denticulada; Fruto não observado.

Distribuição geográfica: A. brevipes ocorre do norte da América do Sul até o Brasil (Govaerts, 2015). No Brasil, ocorre em vários estados (Pará, Roraima, Bahia, Maranhão, Mato Grosso, Rio de Janeiro, São Paulo e Paraná) e em três domínios fitogeográficos (Amazônia, Cerrado e Mata Atlântica) (Barros et al., 2015). Contudo, apesar da ampla distribuição, poucos e esparsos registros são conhecidos no Brasil, de acordo com os registros disponíveis na rede speciesLink (CRIA, 2015).

Material examinado: BRASIL. São Paulo: Botucatu, Cachoeira da Marta, 850 m. s.n.m., 2255'54'S 48'24'26'W, X-2013, G. M. Marcusso \& P. R. Sanine 130 (HRCB).

Fenologia: Foi observado com flores de novembro a fevereiro. 


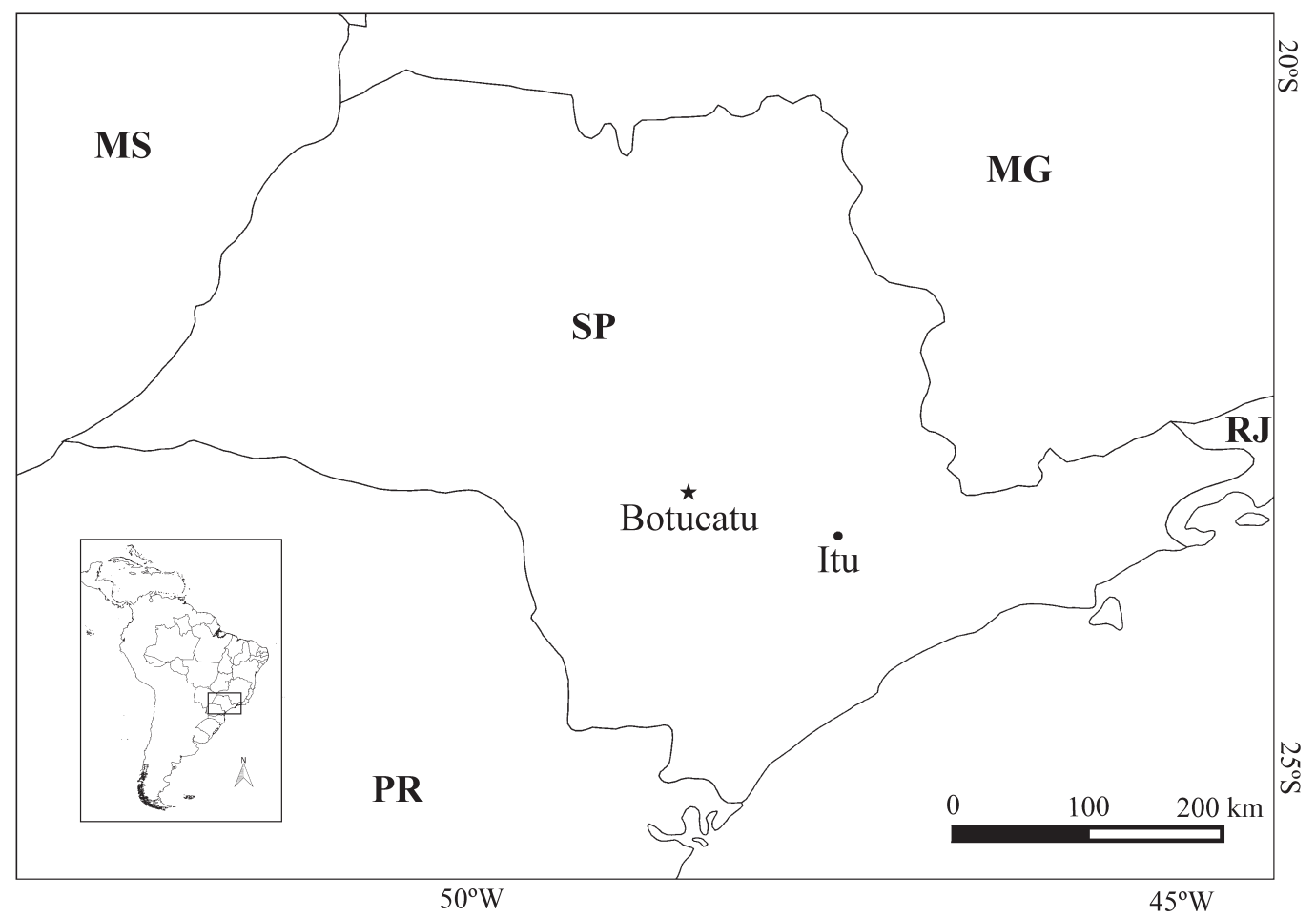

Fig. 1. Localização dos registros de Anathallis brevipes no Estado de São Paulo. Estrela: local da redescoberta; Círculo: local do primeiro registro no estado. (MG: Minas Gerais; MS: Mato Grosso do Sul; PR: Paraná; SP: São Paulo; RJ: Rio de Janeiro).

Notas taxonômicas: Há registros de duas outras espécies desse gênero com ocorrência simpátrica na região (Ferreira et al., 2010; Ferreira et al., 2013). Anathallis marginata (Barb. Rodr.) F. Barros \& Barberena, recentemente registrada no Estado de São Paulo (Ferreira et al., 2013), que se distingue de $A$. brevipes pelo menor porte da planta e pela inflorescência que ultrapassa a altura das folhas e A. obovata (Lindl.) Pridgeon \& M. W. Chase, que se distingue facilmente de $A$. brevipes pelas folhas obovadas e pela inflorescência multiflora.

Conservação: Com esse registro, o status de $A$. brevipes, que era considerada como presumivelmente extinta (EX) no Estado de São Paulo, segundo os critérios da IUCN utilizados por de Mamede et al. (2007), por não haver coletas por mais de 50 anos, deve ser considerado agora como vulnerável (VU), pois ainda corresponde a alguns critérios.
Comentários ecológicos: A população apresentava hábito epifítico na área da coleta. Foi encontrado próximo a uma cachoeira, em Floresta Estacional Semidecídua de encosta, local com abundante umidade atmosférica e luminosidade, a cerca de 850 metros de altitude. Aparentemente, é uma espécie rara na localidade, foram observados poucos indivíduos em um único forófito.

O presente registro, além de colaborar com o conhecimento sobre a distribuição do táxon, irá contribuir para o projeto em andamento da Flora Fanerogâmica do Estado de São Paulo, assim como outras recentes redescobertas e novos registros feitos no estado (e.g. Miranda \& Menini Neto, 2010; Ferreira et al., 2013; Miranda \& Menini Neto, 2014; Miranda et al., 2014). Tais redescobertas demonstram as lacunas de conhecimento sobre a flora do estado, sobretudo quando se trata de plantas de tamanho reduzido, fato que dificulta a observação em campo (Miranda \& Menini Neto, 2010). 


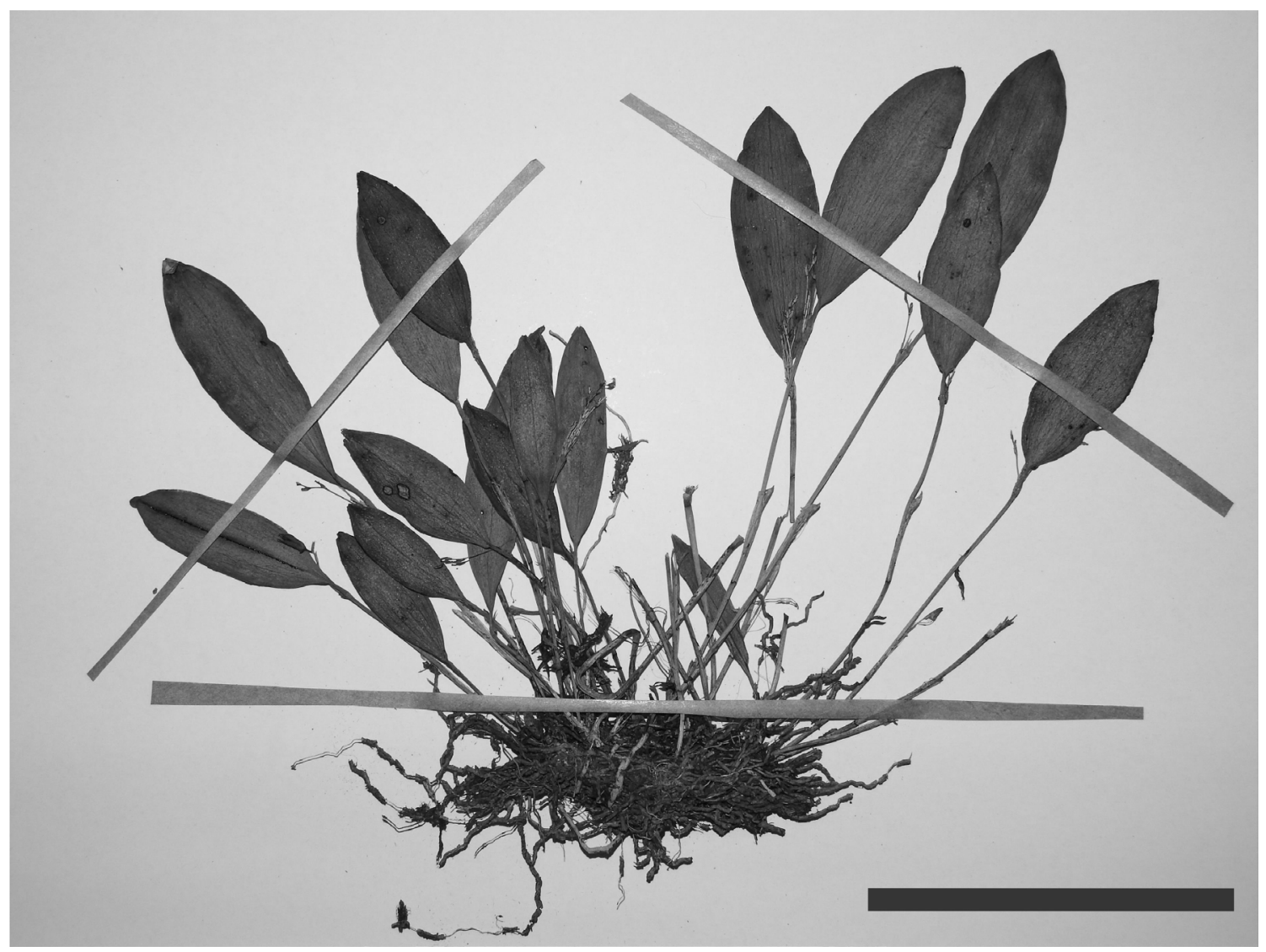

Fig. 2. Anathallis brevipes (G.M. Marcusso \& P.R. Sanine 130, HRCB). Escala: 6 cm.

\section{Agradecimentos}

Ao Dr. Diogo Araújo e aos dois revisores anônimos pelas valiosas sugestões no manuscrito.

\section{Bibliografia}

BARROS, F. 2007. A família Orchidaceae na Flora do Estado de São Paulo e suas espécies ameaçadas. En MAMEDE, M. C. H., V. C. SOUZA, J. PRADO, F. BARROS, M. G. L. WANDERLEY. \& J. G. RANDO (org.). Livro Vermelho das Espécies Vegetais Ameaçadas do Estado de São Paulo, pp. 47-52. Instituto de Botânica, São Paulo, SP.

BARROS, F. DE, F. VINHOS, V. T. RODRIGUES, F. F. V. A. BARBERENA, C. N. FRAGA, E. M. PESSOA, W. FORSTER, L. MENINI NETO, S. G. FURTADO, C. NARDY, C. O. AZEVEDO \& L. R. S. GUIMARÃES. 2015. Orchidaceae. En Lista de
Espécies da Flora do Brasil. Jardim Botânico do Rio de Janeiro. Disponível em: <http://floradobrasil.jbrj. gov.br/jabot/floradobrasil/FB37204>. Acesso em: 16 julho 2015

CEPAGRI 2015. Centro de Pesquisas Meteorológicas e Climáticas Aplicadas a Agricultura. Disponível em: http:/www.cpa.unicamp.br/outras informacoes/ clima_muni_457.html. Acesso em: 10 de agosto 2015.

CHIRON, G. \& R. X. BOLSANELLO. 2015. As orquídeas da Serra do Castelo (Espírito Santo, Brasil). Contribuição à flora de orquídeas do Espírito Santo volume 1. Tropicalia, Voreppe. $371 \mathrm{pp}$.

CRIA 2015. Centro de Referência em Informação Ambiental. Disponível em: http://www.cria.org.br. Acesso em 16 julho 2015.

FERREIRA, A. W. C., M. I. S. LIMA \& E. R. PANSARIN. 2010. Orchidaceae na região central de São Paulo, Brasil. Rodriguésia 61: 243-259.

FERREIRA, A.W.C. D. H. BAPTISTA \& E. R. PANSARINM. 2013. Anathallis marginata (Orchidaceae, Pleurothallidinae): a new Record from the state of São Paulo, Brazil. Richardiana 13: 156-163. 
GOVAERTS, R. 2015. World Checklist of Orchidaceae. Facilitated by the Royal Botanic Gardens, Kew. Published on the Internet; http://apps.kew.org/ wcsp/ Acesso em: 16 Julho 2015.

INSTITUTO BRASILEIRO DE GEOGRAFIA E ESTATÍSTICA. 2012. Manual técnico da vegetação brasileira. IBGE, Coordenação de Recursos Naturais e Estudos Ambientais, 2 ${ }^{\text {a }}$ ed., Rio de Janeiro. $276 \mathrm{pp}$.

MAMEDE, M.C.H., V. C. SOUZA, J. PRADO, F. BARROS, M. G. L. WANDERLEY \& J. G. RANDO (org.). 2007. Livro Vermelho das Espécies Vegetais Ameaçadas do Estado de São Paulo. Instituto de Botânica, São Paulo, SP. 165 pp.

MIRANDA, M.R. \& L. MENINI NETO. 2010. Redescoberta de Centroglossa macroceras Barb. Rodr. e Thysanoglossa jordanensis Porto \& Brade (Orchidaceae), duas micro-orquídeas consideradas presumivelmente extintas no Estado de São Paulo, Brasil. Hoehnea 37: 403-405.

MIRANDA, M.R. \& L. MENINI NETO. 2014. New
Record in the Brazilian Atlantic Forest: Specklinia aristata (Orchidaceae: Pleurothallidinae). Richardiana 14: 198-204.

MIRANDA, M.R., L. MENINI NETO, F. J. JESUS \& G. R. CHIRON. 2014. Anathallis guarujaensis (Orchidaceae, Pleurothallidinae): redécouverte d'une espèce considérée comme éteinte. Richardiana 14: 169-175.

MYERS, N., R. A. MITTERMEIER, C. G. MITTERMEIER, G. A. B. DA FONSECA \& J. KENT. 2000. Biodiversity hotspots for conservation priorities. Nature 403: 853-858.

PONÇANO, W.L., C. D. R. CARNEIRO, C. A. BISTRICHI, F. F. M. ALMEIDA. \& F. L. PRANDINI. 1981. Mapa Geomorfológico do Estado de São Paulo. São Paulo: Instituto de Pesquisas Tecnológicas. 94 pp.

PRIDGEON, A.M., M. W. CHASE, P. J. CRIBB \& F. N. RASMUSSEN. 2005. Genera Orchidacearum vol. 4, Epidendroideae (Part 1). Oxford University Press, United Kingdom.

Original recibido el 16 de septiembre de 2015; aceptado el 15 de febrero de 2016. 
